\title{
Cuckoo Search based Optimization for Multimodal Biometrics (Signature, Speech and Palmprint)
}

\author{
Harjinder Kaur \\ Research Fellow \\ SGGSWU, Fatehgarh Sahib, Punjab.
}

\author{
Gaganpreet Kaur \\ Assistant Professor \\ SGGSWU, Fatehgarh Sahib, Punjab.
}

\begin{abstract}
Biometric system based on unimodel biometrics are not able to meet the desired performance requirements for security purpose due to problems such as intra-class variations, noisy data, unacceptable error rates and low performance. Multimodal biometrics refers to the use of two or more biometrics modalities in a single identification system. The combination of different modalities is used to improve the recognition accuracy. In this proposed work, for identification based multimodal offline signature verification system, speech verification system and palmprint are combined to enhance the security and accuracy. Signature and speech are naturally produced and palmprint cannot be changed or lost. SIFT is used to extract the features of offline signature, MFCC is used to extract the features of speech and ASM is used to extract the features of palmprint. Using a technique named sum rule uses fusion at feature level in this work. To enhance the accuracy the nature based Cuckoo Search Algorithm is used to extract the best values as optimization technique. The experimental results demonstrated that the proposed multimodal biometrics system achieves a recognition accuracy of $100 \%$ with false rejection rate (FRR) of $=0.0 \% \&$ false acceptance rate (FAR) of $=0.0 \%$.
\end{abstract}

\section{Keywords}

Multimodal Biometrics, Scale invariant feature transform (SIFT), Mel Frequency Cepstral Coefficient (MFCC), Active Shape Model (ASM), Feature level Fusion, Cuckoo Search(CS) Algorithm.

\section{INTRODUCTION}

A biometric characteristic is a general term used to describe a measurable physiological and/or behavioral characteristic that can be used for automated recognition. Biometric authentication is one of the most exciting technical improvements of recent history and looks set to change the way in which the majority of individuals live, Security. The prominence of biometrics has addressed the problems that overcome traditional verification methods. Biometrics signifies the automatic identification or verification of an individual by using certain physiological or behavioral traits associated with the person. A multimodal biometric system combines two or more features of a person to be recognize together to determines a person's authentication. The fusion of multiple modalities can provide complementary information and increase the accuracy of the overall decisionmaking process. In this paper, we describe a multimodal biometric system based on palm print; speech and signature features are combined as these modalities are widely accepted. The proposal of applying the cuckoo search algorithm is one of the recent optimization algorithm in the league of nature based algorithm.

\section{DESIGN AND IMPLEMENTATION}

\subsection{Proposed Work}

In this work, an offline signature verification system, speech verification system and palmprint are combined as these modalities are widely accepted. Scale invariant feature transform(SIFT)algorithm is used for feature extraction of signature, key points are detected at multiple scales. Mel Frequency Cepstral Coefficients(MFCC) algorithm[7] is used for feature extraction of speech, which takes the human perception sensitivity with respect to frequencies and reduces the blurrness of spectral and active Shape Model(ASM)[4] is used for feature extraction of palmprint, which locates the landmark points of any statistically image. Then these extracted features from speech, signature and palmprint are fused at feature level using sum rule. Feature vectors are firstly fused with sum method but to add these different dimensional vectors could not be possible. So, padding method can be used where 0 can be added to the end of the lower dimensional feature vector. After fusion, cuckoo search is applied to extract optimized features. 


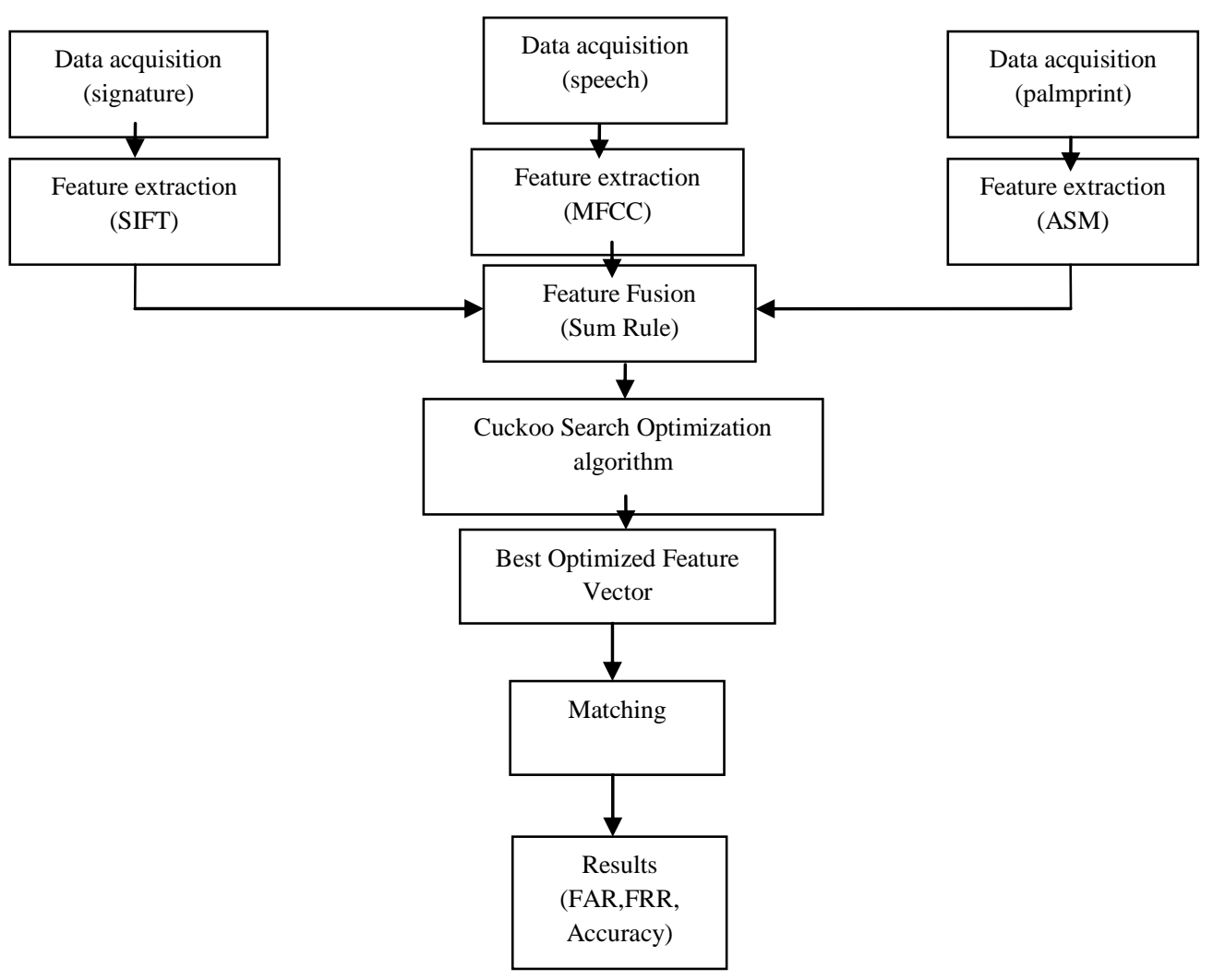

Fig. 1 Flow Diagram

\subsection{Data Acquisition}

A database of 75 samples (speech, signature and palm print) consisting of a Training set and Testing set is used. Training Set consists of 75 genuine samples from 25 known users. Each of the users contributes 3 samples for speech, signature and palm print.

1.) Signature recognition:In this proposed work, we deal with an Off-line signature recognition technique, where the signature is captured and shows in the format of image only. The offline signatures samples are collected by users are stored in .jpg file format. We use the SIFT to extract the parameters of signature and then verified the signature. In SIFT, scale spaces are construct by difference of Gaussians(DOG) and locate the maxima and minima of DOG as keypoints.Filter the edges and low contrast edges are discarded.Orientations are assign to localized keypoints and build the keypoints descriptors and stored as extract of features.



Fig.2
2.) Speech recognition:Speaker recognition is the task of recognizing speakers using their speech.The biometric system using speech analyzes and extracts speakerspecific features from the speech signal. There are multiple equipments to store the different types of voices ,but in this work data is collected from www.webcrawler.com and stored in .wav format. There are two types of datafor speaker recognition text dependent and text independent. Text independent data is used in the present work. For the speech signal, Mel Frequency Cepstral Coefficients (MFCC) are computed. MFCCs are mean and standard deviation normalized using normalization values computed on the speech part of thedata.

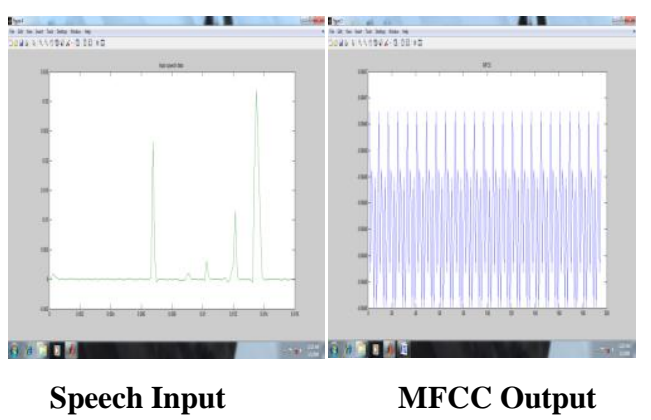

Fig 3

3.) Palm Print Recognition: In this work, Active Shape Model is used to extract the features of palmprint which locates the landmark points(set of points) of the image.Active Shape Model has a 2-stage loop: in the first stage, given the initial labels, searching 
for a newposition for every label point in its local region which best fits the corresponding texture model; in the second stage, updating the shape parameters which best fits these newlabel positions[1].

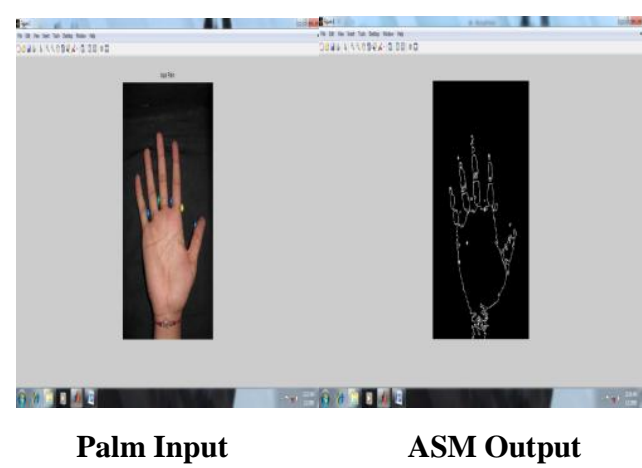

Fig. 4

\subsection{Fusion for Feature Vectors}

In our work, 128 no. of feature vector can be generated by SIFT algorithm for offline signature,195 no. of feature vector can be generated by MFCC for speech samples and $300 \times 240$ no. of feature vector can be genrated by ASM for palmprint samples. The sum rule is used to fuse these feature vectors, butto add these different dimensional vectors could not be possible. So, padding method can be used where 0 can be added to the end of the lower dimensional feature vector[19]. After Padding, fuse these three feature vectors, firstly the sum rule is apply on signature and speech feature vectors and after that which feature vector is obtain from these two vectors that is fused with the feature vector of palmprint as to obtain a single fused matrix.

\subsection{Optimization}

Cuckoo Search Optimization(CSO) is a population based algorithm. This optimization algorithm isintroduced which is inspired by the lifestyle of a bird family called cuckoo.Basically, CS is a population oriented stochastic global search algorithm that is mainly used for finding a global optimal solution. It is observed that the quality or fitness of a solution can easily be proportional to the value of objective function.CS optimization method is a nature inspiredalgorithm, which is based on the obligate brood parasitic behaviorof some cuckoo species by laying their eggs in the nests of other host birds (of other species). Some host birds can engage direct conflict with the host birds., if a host bird discovers the eggs are not their own, it will either throw these alien eggs away or simply abandon its nest and build a new nest elsewhere[8].

There are three idealized rules in the cuckoo search algorithm are:

1. Each cuckoo lays one egg at a time and dumps it in a randomly chosen nest.

2. The best nests with high quality of eggs will carry over to the next generation.

3. The number of available host nest is fixed, and the egg laid by a cuckoo is discovered by the host bird with a probabiliy of $\mathrm{pa}[0,1]$.

Table 1 Cuckoo Search Optimization Algorithm

\section{CSO_Algorithm}

Initialize parameters

No.of nests $=$;

Discovery rate of alien eggs/solutions

$\mathrm{Pa}=0.25 \%$

Tolerence

Tol=1.0e-5;

Simple bounds of search domain

Lower bound $\mathrm{Lb}$;

Upper bound Ub;

Step I: Generate an initial population of $\mathrm{n}$ host nests

for $\mathrm{i}=1: \mathrm{n}$

$\operatorname{nest}(\mathrm{i}: 1)=\mathrm{Lb}+(\mathrm{Ub}-\mathrm{Lb})+\operatorname{rand}(\operatorname{size}(\mathrm{Lb}))$;

end

Step II: Calculate the fitness value by using

Fitness $\left.(\mathrm{y})=\operatorname{mean}(\mathrm{x}) * \operatorname{size}(\mathrm{x}, 1)+\operatorname{sum}\left(\mathrm{x}^{2}-10 \cos (2 \pi \mathrm{x}), 1\right)+\operatorname{sum}\left(-\mathrm{x}^{1 / 2}\right), 1\right) ;$

Step III: Find the best nest from whole nest by using fitness value.

Step IV: Start iterations 
N_iter = 0;

While(fitness $>$ Tol)

Evaluate the cuckoos from best nest by using levy flights and generate new solution.

Remove the empty nests from the new solution and generate a new nest.

Get the best values from new nest.

Update Counter

N_iter $=$ N_iter+n;

end

Step V: Return best solutions.

\section{RESULTS}

The performance of the system is analyzed from the time graph and by calculating FAR and FRR of the system and then accuracy is calculated. FAR for the proposed system is $0 \%$ and FRR is also $0 \%$ and accuracy is $100 \%$. Therefore, accuracy of the system comes out to be $100 \%$.

Table 2 False Rejectance Rate

FRR for Palm, Signature \& Speech Samples:

Total Number of Samples in the database $=75$

Number of Sample that falsely rejected $=00$

FRR $=\frac{\text { Number of Samples that Falsely rejected }}{\text { Total Number of Samples }}$

So, $\mathrm{FRR}=0 / 75=0$

Table 3 False Acceptance Rate

FAR for Palm, Signature \& Speech Samples:

Total Number of Samples in the database $=75$

Number of Sample that falsely accepted $=00$

FAR $=\frac{\text { Number of Samples that Falsely accepted }}{\text { Total Number }}$

So, $\mathrm{FAR}=0 / 75=0$

Table 4 Accuracy

Accuracy $=100-($ FAR+FRR $) \%$

Accuracy $=100-(0.00+0.00) \%$

Accuracy $=100 \%$

Fig. 2 shows the comparison of Previous \& Proposed techniques that results that our proposed technique gives more accurate results than the previous. The accuracy of the system is approximately $98.4 \%$ which is better than the other Systems.

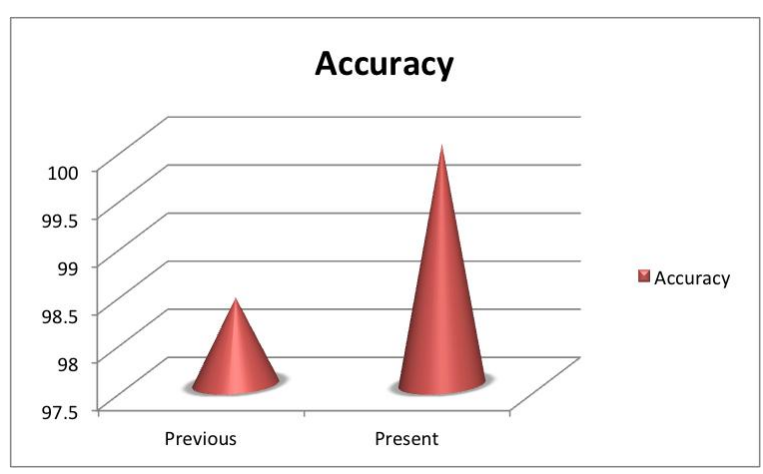

Fig 5 Comparison of Previous \& Proposed Technique

\section{CONCLUSION \& FUTURE WORK}

The performance of single modality based biometric recognition has been suffering from the different noisy data, non-universality of biometric data, and susceptibility of spoofing. This paper specifies at feature level fusion of multimodal biometric system as it gives the better results than single biometric trait. The cuckoo search algorithm is one of the recent optimization algorithm which is used for finding and short listing the features. This proposed method decreased the FAR as well as FRR, \& has increases the system performance on the given data set. The proposed multimodal biometrics system achieves a recognition accuracy of $100 \%$ and proved that it is more efficient and accurate.

Future works could go in the direction of using more robust techniques and any other fusion level can be used for different modalities. Also, the system should be tested on a larger database to validate the robustness of the model.

\section{REFERENCES}

[1] Lu, Lu., Xiaoxun, Zhang., Youdong, Zhao., Yunde, Jia, 2006."Ear Recognition based on Statstical Shaped Model", proc. of the First International Conference on Innovative Computing, Information and Control (ICICIC'06) 0-7695-2616- IEEE.

[2] Kartik, P., Prasad, Prasana, Mahadeva., 2008."Noise Robust Multimodel Biometric Person Authentication System using Face, Speech and Signature Features" proc. of IEEE,pp.978-1-4244-2746.

[3] Rodriguez., Puente., Crespo Garacia, A., Lara, poza., Mezcua, Rui.z, 2008."Study of Different Fusion Techniques for Multimodal Biometric Authentication" proc. of IEEE, pp.666-671. 
[4] Jang, Changhyuk., Jung, Keechul., 2008."Human Pose Estimation using Active Shape Model", proc. of World Academy of Science Engineering and Technology.

[5] Hu, Junlin., Xue, Yanxue., Zhao, Yongwei., Lu, Jiwen, 2008."Palmprint Recognition Based on Multiple Feature Information Fusion" proc. of IEEE, 978-1-4244-2179$4 / 08$.

[6] Kisku, Ranjan, Dakshina., Rattani, Ajita., Gupta, Phalguni., Sing, Kanta, Jamuna., "Biometric Sensor Image Fusion for Identity Verification: A Case Study with Wavelet-based Fusion Rules and Graph matching" proc. of IEEE 978-1-4244-4179-2/09.

[7] Kaur, Mandeep., Girdhar, Akshay., Kaur, Manavjeet., 2010. "Multimodal Boimetric system using Speech and Signature modalities" Proc. Of International journal of computer applications, vol. 5-No.12, pp. 13-16.

[8] Yang, X,S., Deb, S., 2010.“Engineering Optimization by Cuckoo Search" proc. of Int.j.Mathematical modeling and numerical optimization, vol. 1, No.4, 330-343.

[9] Kim, Dong-Ju., Chung, Kwang-Woo., and Hong, Kwang-Seok., 2010."Person Authentication using Face, Teeth and Voice Modalities for Mobile Device Security", proc. of IEEE, vol. 56, No. 4, pp. 2678-2685.

[10] Elumalai, K., Kannan, M., Soliman, Hassan., 2011."Multimodal Authentication for High End Security" Proc. of International Journal on Computer Science and Engineering (IJCSE) ISSN: 0975-3397, vol. 3 No. 2.

[11] Y, K, Rajput., Melissa, Amanna., Mankhush, Jagwat., Mayank Sharma, 2011."Palmprint Recognition using Image Processing" in proc. of vol. 3 ISSN (09743-3375).

[12] Nagar, Abhishek., Nandakumar, Karthik., Jain, K, Anil., 2012."Multibiometric Cryptosystems Based on FeatureLevel Fusion"proc. of IEEE Transactions on Information Forensics and Security, vol.7,No.1.

[13] Tiwari, Vipinkumar, 2012. "Face Rocognition based on Cuckoo Search algorithm", proc. of Indian Journal of
Computer Science and Engineering ISSN: 0976-5166, vol. 3 No.3

[14] Nakagawa. Seiichi., wang, Longbia.., Ohtsuka, Shinji., 2012." Speaker Identification and Verification By combining MFCC and Phase Information" in proc. of IEEE Transactions on Audio, Speech, and Language vol. 20,No. 4.

[15] Patil, B, Savitri 2012. "A Study of Biometric, Multimodal Biometric Systems: Fusion Techniques, Applications and Challenges" IJCST vol. 3, Issue 1.

[16] Kaur, Sandeep., Kaur, Gaganpreet, 2013. "Optimized Palm Recognition using Cuckoo search Algorithm", proc. of International Journal of Advance Research in Computer and Engineering vol. 2, Issue 11.

[17] Kaur, Harjinder., Bhatial, kaur, Amandeep., 2013. "Security and Privacy in Biometrics: A Review", proc. of vol.1, Isssue-2, ISSN No. 2320-7639.

[18] Sood, Monica., Kaur, Gurline., 2013. "Speaker Recognition Based On Cuckoo Search Algorithm" proc. of International Journal of Innovative Technology and Exploring Engineering (IJITEE) ISSN: 2278-3075, vol.2, Issue-5.

[19] Kaur, Dapinder., Kaur, Gaganpreet., Singh, Dheerendra., 2013."Efficient and Robust Multimodel biometric System for Feature level fusion (Speech and signature)" proc. of International Journal of Computer applications (0975-8887) vol. 75- N0.5.

[20] Jain, K, Anil., Hong,L., Kulkarni,Y., "A Multimodal Biometric System using Fingerprint, Face and Speech" in proceedings of second international conference on AVBPA,pp. 182-187.

[21] Solliman, Hassan., Mohamed, Saber, Abdelnasser., Atwan, Ahmed, "Feature level Fusion of Palm veins and signature Boimetrics" in proc. of video and image processing and network security, vol. 12 No.01 28. 